\title{
Center of mass acceleration feedback control for standing by functional neuromuscular stimulation: A simulation study
}

\author{
Raviraj Nataraj; ${ }^{*}$ Musa L. Audu, PhD; ${ }^{1}$ Robert F. Kirsch, PhD; ${ }^{2}$ Ronald J. Triolo, PhD $^{\mathbf{1}}$ \\ ${ }^{1}$ Motion Study Laboratory, Louis Stokes Cleveland Department of Veterans Affairs Medical Center, Cleveland, OH; \\ ${ }^{2}$ Department of Biomedical Engineering, Case Western Reserve University, Cleveland, $\mathrm{OH}$
}

\begin{abstract}
The potential efficacy of total body center of mass (COM) acceleration for feedback control of standing balance by functional neuromuscular stimulation (FNS) following spinal cord injury (SCI) was investigated. COM acceleration may be a viable alternative to conventional joint kinematics because of its rapid responsiveness, focal representation of COM dynamics, and ease of measurement. A computational procedure was developed using an anatomically realistic, threedimensional, bipedal biomechanical model to determine optimal patterns of muscle excitations to produce targeted effects upon COM acceleration from erect stance. The procedure was verified with electromyographic data collected from standing nondisabled subjects undergoing systematic perturbations. Using 16 muscle groups targeted by existing implantable neuroprostheses, we generated data to train an artificial neural network (ANN)-based controller in simulation. During forward simulations, proportional feedback of COM acceleration drove the ANN to produce muscle excitation patterns countering the effects of applied perturbations. Feedback gains were optimized to minimize upper-limb (UL) loading required to stabilize against disturbances. Compared with the clinical case of maximum constant excitation, the controller reduced UL loading by $43 \%$ in resisting external perturbations and by $51 \%$ during simulated one-arm reaching. Future work includes performance assessment against expected measurement errors and development of user-specific control systems.
\end{abstract}

Key words: acceleration, balance, center of mass, control system, feedback, functional neuromuscular stimulation, neuroprosthesis, posture, rehabilitation, spinal cord injury, standing.

\section{INTRODUCTION}

This study investigated using acceleration of total body center of mass (COM) as alternative feedback to conventional joint kinematics for continuously adjusting stimulation to muscles following spinal cord injury (SCI) to maintain stable standing against perturbations to postural balance. Neuroprostheses employing functional neuromuscular stimulation (FNS) have effectively restored basic standing function following SCI using preprogrammed stimulation to facilitate sit-to-stand maneuvers and continuous, constant stimulation to maintain upright posture [12]. Because stimulation is applied at constant levels to maintain standing, the user is required to exert significant upper-limb (UL) effort on an assistive device (e.g., walker) to stabilize against postural disturbances. Sustained UL

Abbreviations: ANN $=$ artificial neural network, AP $=$ anteriorposterior, $\mathrm{BOS}=$ base of support, $\mathrm{BW}=$ body weight, $\mathrm{COM}=$ center of mass, $\mathrm{DF}=$ dorsiflexion, $\mathrm{DOF}=$ degree of freedom, EMG = electromyography, FAS $=$ feasible acceleration sets, FNS = functional neuromuscular stimulation, FTP = functional task performance, $\mathrm{ML}=$ medial-lateral, $\mathrm{PD}=$ proportionalderivative, $\mathrm{PF}=$ plantar flexion, $\mathrm{PID}=$ proportional-integralderivative, $\mathrm{SCI}=$ spinal cord injury, $\mathrm{SD}=$ standard deviation, $\mathrm{UL}=$ upper limb.

*Address all correspondence to Raviraj Nataraj; Louis Stokes VAMC, 10701 East Blvd, Room C-15 (Motion Study Laboratory), Cleveland, OH 44106; 216-791-3800, ext 3838. Email: $\underline{\text { rxn25@case.edu }}$

http://dx.doi.org/10.1682/JRRD.2010.12.0235 
effort compromises the utility of standing with FNS by limiting reach and manual function and reduces standing time by expediting the onset of upper-body fatigue.

Standard joint angle feedback has been extensively investigated for closed-loop control of standing with FNS. It has been implemented in isolation for individual joints, including the knees [3-4], hips [5-6], and ankles [7-8]. These studies showed measures of improvement in disturbance response but effectively constrained the standing system to single planes of movement. Comprehensive (ankles, knees, hips, and trunk) three-dimensional control of standing with FNS based on joint feedback has been investigated in simulation [9]. Although it significantly reduced UL effort during postural perturbations when compared with constant, maximal stimulation, this system required tuning 18 separate gain parameters for the proportional-derivative (PD) feedback from 9 individual joints. This required instrumentation at each joint under active control, which may be cumbersome and impractical for routine clinical deployment. Furthermore, in order to effectively compensate for the delay between stimulus onset and peak muscle force generation, standard PD joint feedback gains may be undesirably high, leaving the control system prone to instability.

Acceleration has been previously suggested as an effective means for assessing balance [10-12] and offers several potential advantages over joint-based control of standing with FNS. First, it is sensitive to the inertial effects of rapidly acting perturbations and can respond before significant changes in standing posture can occur, thereby providing a more potent initial feedback signal than position-based control. Acceleration of the system COM provides a representation of global system dynamics that are critical for standing control [13]. Finally, adequate measurement of COM acceleration may be plausible with only a few well-placed accelerometers. This is because perturbed standing can be represented with a minimal number of synergies [14-15] and nearly 75 percent of body mass is concentrated centrally across the pelvis, abdomen, and trunk [16].

The primary objective of this study was to develop and evaluate, in simulation, a feedback control system for FNS standing that uses gain-modulated COM acceleration inputs to produce optimal muscle excitation patterns that counter the effects of postural disturbances. We employed a model-based approach to determine the feasibility and basic operating characteristics of the controller prior to online testing with subjects with SCI. The controller con- sisted of using proportional COM acceleration feedback to drive an artificial neural network (ANN). We trained this ANN on muscle excitation patterns optimized to produce target changes in COM acceleration from the neutral, erect standing posture. To validate the optimal acceleration-excitation synergy represented by the data used to train the ANN, we collected electromyographic (EMG) data during systematic perturbation of nondisabled standing subjects. We compared the COM acceleration directions in which certain muscle groups were most active following a perturbation from neutral standing across both data sets. We evaluated controller performance according to the reduction in UL effort necessary to stabilize the model against disturbances with active controller modulation of muscle excitation levels compared with the case of constant excitation levels analogous to clinical stimulation paradigms.

\section{METHODS}

The overall system included two parallel controllers (FNS muscle control and UL loading) acting on a threedimensional model of SCI bipedal standing ("ThreeDimensional Model of Spinal Cord Injury Stance” section) to maintain an erect, neutral set point position (Figure 1). We defined the set point as a single reference position that the control system was designed to maintain. We selected the most erect posture corresponding to the highest vertical COM position above the center of the base of support (BOS) as the desired set point for the model. The FNS control system employed negative feedback of measured COM acceleration changes, thereby driving an ANN to modulate muscle excitation levels to counter the effects produced by postural disturbances. We represented volitional UL loading by proportional-integral-derivative (PID) control of the shoulder position ("Upper-Limb Controller" section) corresponding to the set point. The objective of both the FNS and UL control systems was to resist disturbances imposed on the standing model while in the set point posture. We evaluated the FNS controller according to the reduction in shoulder position controller output (i.e., reduction in UL loading) under various postural disturbances using feedback controller modulation of the muscle excitation levels compared with the constant muscle excitation levels described in the "Determining Optimal and Maximal Sets of Constant Excitation Levels for Baseline Performance" section. 


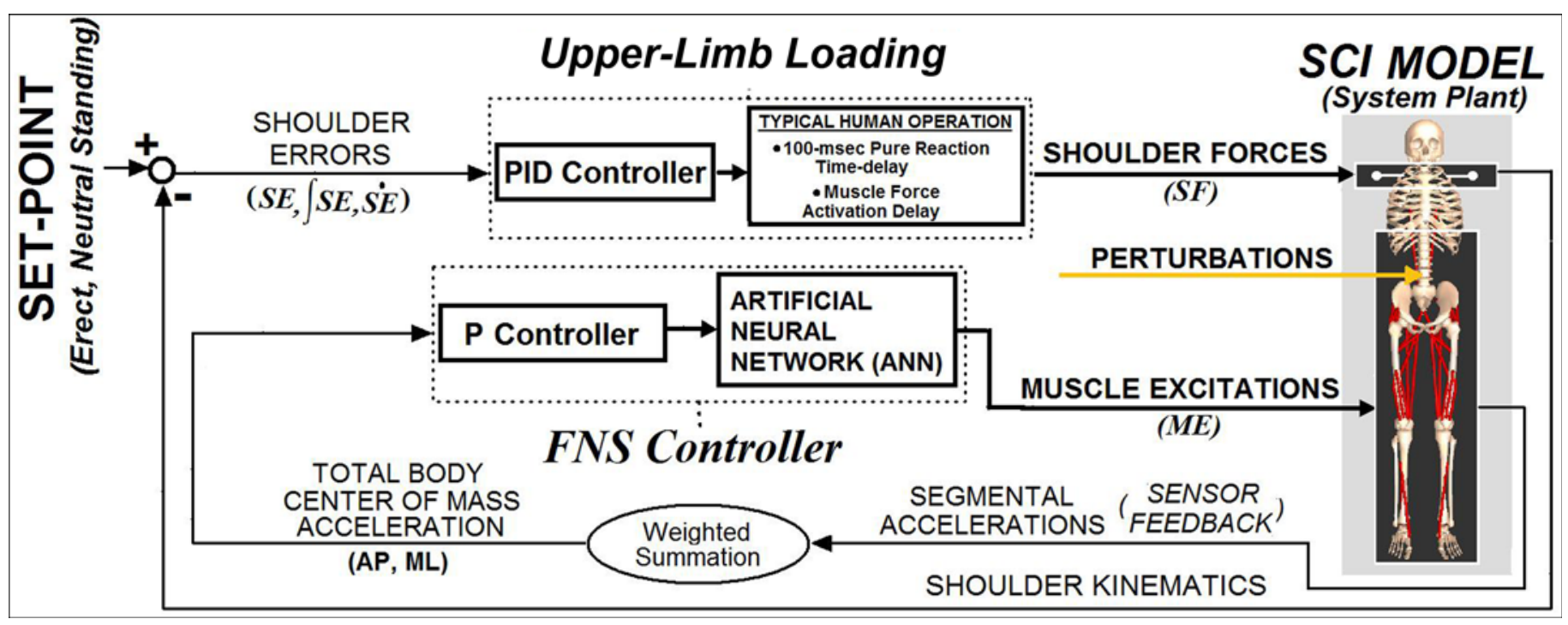

Figure 1.

Overall model system. Two parallel controllers act to maintain three-dimensional (3-D) model of bipedal spinal cord injury stance at set point position against postural perturbations. (1) Functional neuromuscular stimulation (FNS) controller modulates trunk and lower-limb muscle excitations according to center of mass (COM) acceleration feedback in anterior-posterior (AP) and medial-lateral $(\mathrm{ML})$ directions driving artificial neural network (ANN). ANN is trained to output muscle excitation changes that counter measured effects induced by disturbances and net recovery responses upon COM. (2) Upper-limb (UL) controller, representing user volitional loading, produces 3-D point forces at shoulders according to position errors relative to shoulder set point posture. COM acceleration and shoulder positional errors are expressed in globally fixed 3-D Cartesian coordinates. Gains for UL control are determined according to Ziegler-Nichols tuning rules. FNS controller gains are optimized using global-search algorithm to minimize UL controller output ("Ioading") against perturbations. $\mathrm{ME}=$ muscle excitations, $\mathrm{P}=$ proportional gain, $\mathrm{PID}=$ proportional-integral-derivative, $\mathrm{SCI}=$ spinal cord injury, SE = shoulder errors, SF = shoulder forces.

In creating the data space that governs FNS controller action, the model determined instantaneous changes in COM acceleration induced across the anterior-posterior (AP) and medial-lateral (ML) dimensions by changes in activation level from the set point stance for each muscle group available for FNS control. It then formulated optimal patterns of muscle activation ("Procedure for Creating Optimal Muscle Activation Data According to Center of Mass Acceleration in Targets” section) to produce target changes in COM acceleration about the erect set point position that were feasible subject to force-generating capabilities of the included muscle groups. This is similar to the concept of "induced accelerations" introduced in Zajac and Gordon [17] to determine the net effect of changes in muscle activation on joint angular accelerations given a particular system state. We validated this model-based optimization procedure for coordinating muscle activity according to changes in COM acceleration using the EMG data collected from nondisabled individuals undergoing disturbances while standing ("Collection of Electromyographic Data of Nondisabled Individuals During Perturbed Bipedal Standing” section). We compared the net (across AP and ML dimensions) COM acceleration directions along which muscle groups were most active between the EMG and model-based data ("Comparing Nondisabled Electromyographic Data Against Optimal Model-Based Data” section).

We applied the optimization procedure for producing optimal changes in muscle activation in accordance with targeted changes in COM acceleration from erect stance to create data representing a synergy used to train the ANN ("Creating Artificial Neural Network for Functional Electrical Stimulation Control” section). Each two-dimensional (AP, ML) COM acceleration target represented a single training point of inputs, and the corresponding optimal excitation levels represented a single training point of out- 
puts. For the purposes of ANN training, we assumed muscle activation, the muscle state variable determining force output level, to be directly proportional to excitation, the actual control input and analog for FNS stimulation level, for ANN training. We subsequently addressed excitationactivation coupling during forward simulations with specified perturbations ("Perturbation Simulations" section) with optimal tuning of the feedback controller gains ("Tuning Center of Mass Acceleration Feedback Controller” section) to minimize UL loading in the presence of activation dynamics [18]. We observed control system performance during resistance of disturbances under two-arm and onearm support conditions and during simulated one-arm reaching and manipulation of a weighted object ("Testing Controller Performance” section). Nataraj et al. originally described the models for SCI bipedal standing and volitional UL loading, determination of baseline excitation levels, and test perturbations [9].

\section{Three-Dimensional Model of Spinal Cord Injury Stance}

We developed a three-dimensional computer model of human bipedal stance in SIMM (Software for Interactive Musculoskeletal Modeling, MusculoGraphics, Inc; Santa Rosa, California), adapted from a previously described representation of the lower limbs [19] and trunk [20]. This model consisted of 9 segments (2 feet, 2 thighs, 2 shanks, pelvis-lumbar component, and head-arm-trunk complex) with 15 anatomical degrees of freedom (DOFs) representing bilateral motions of ankle plantar flexion (PF) and dorsiflexion (DF), ankle inversion and eversion, knee flexion and extension, hip flexion and extension, internal rotation and external rotation, hip abduction and adduction, and trunk roll-pitch-yaw. We included passive moment properties caused by SCI at these DOFs [21]. Both feet were in constant contact with the ground, defining a closed-chain that effectively reduced the number of independent DOFs to 6 [22]. The lower limbs were in series with a single 3-DOF trunk joint at the lumbrosacral (lumbar 5-sacral 1) region. A total of 58 muscle elements were defined across the trunk and lower limbs. When representing SCI standing by FNS, the only muscle groups actively controlled in the model were consistent with those targeted by the existing 16-channel implanted FNS systems listed in Table 1 [23]. We expect that these implanted systems will be used for individuals with complete thoracic-level SCI for restoring standing balance. We constrained elements within each muscle group to act synchronously at the same level of excitation as if coacti- vated by a single stimulus output at a common motor point (e.g., femoral nerve innervating vasti). Excitation is a normalized quantity (0 to 1 ). We represented muscles as Hill-type actuators with nonlinear force dynamics that included excitation-activation coupling and conventional length-tension and force-velocity properties [18]. We scaled the peak force parameter for each SCI muscle group from nondisabled values to produce the maximum isometric joint moments generated by individuals with complete thoracic-level SCI in response to electrical stimulation [24].

\section{Upper-Limb Controller}

“To approximate [UL] loading that a standing neuroprosthesis user may need to exert on an assistive support to resist postural perturbations, three-dimensional stabilization forces were applied to each shoulder position. PID controller output defined the shoulder force $(S F)$ in each dimension $j$ ([AP, ML], or inferior-superior defined in globally fixed reference frame) according to input shoulder position errors $(S E)$ relative to the reference positions at the set point posture as follows:

$$
S F_{j}=K_{P, j} \times S E_{j}+K_{I, j} \times \int S E_{j}+K_{D, j} \times S \dot{E}_{j},,
$$

where $K_{P}=$ proportional gain, $K_{I}=$ integral gain, and $K_{D}=$ derivative gain [9].

"[UL] controller output acted on shoulder position since the current model does not explicitly include dynamic representations of the arms, which would still otherwise produce reaction loads at the shoulders. The three PID gains $\left(K_{P}, K_{I}\right.$, and $\left.K_{D}\right)$ were determined according to Ziegler-Nichols 2nd method tuning rules [25] against a $100 \mathrm{~N}, 200 \mathrm{~ms}$ forward test pulse at the thorax COM. The same PID gains were used for all three dimensions since only a single Ziegler-Nichols ultimate gain was observed for the single test perturbation. This test pulse induced a model trunk acceleration of $\sim 2.5 \mathrm{~m} / \mathrm{s}^{2}$ which is less than that induced by 'middle level' perturbations [26]. To approximate typical human operator response, $100 \mathrm{~ms}$ pure time delays [27] and muscle force activation delays [18] were applied to the shoulder force outputs. To simulate one-arm support conditions, as required to functionally reach on the contralateral side, only support side shoulder position controller forces were 
Table 1.

Stimulated muscles and corresponding spinal cord injury (SCI) joint moments and baseline excitation levels.

\begin{tabular}{|c|c|c|c|}
\hline $\begin{array}{c}\text { Muscle Group } \\
\text { (Stimulated by Single Channel) }\end{array}$ & Primary Anatomical Actions & $\begin{array}{l}\text { SCI Joint Moment } \\
(\mathrm{N}-\mathrm{m})^{*}\end{array}$ & $\begin{array}{l}\text { Optimal/Maximal } \\
\text { Excitation Level }^{\dagger}\end{array}$ \\
\hline Soleus, Gastrocnemius & Ankle plantar flexion & 55 & $0.049 / 0.262$ \\
\hline Vasti (Medialis, Intermedius, Lateralis) & Knee extension & 80 & $0.960 / 1.000$ \\
\hline Adductor Magnus & Hip extension, hip adduction & 63,30 & $0.767 / 1.000$ \\
\hline Gluteus Maximus & Hip extension & 63 & $1.000 / 1.000$ \\
\hline Semimembranosus & Hip extension, hip adduction & 63,30 & $0.467 / 1.000$ \\
\hline Erector Spinae & Trunk extension & 70 & $0.645 / 1.000$ \\
\hline
\end{tabular}

active” [9]. Nataraj et al. reported and discussed the PID gains [9]. The PID gains produce support loads typically observed in FNS standing systems [28].

\section{Determining Optimal and Maximal Sets of Constant Excitation Levels for Baseline Performance}

"To provide a comparative standard for controller performance across a range of sufficient but constant excitation levels for stable standing, the 'optimal' and 'maximal' muscle excitations (Table 1) were determined for the desired set point posture using the optimizer from [Audu et al.] [29]. The 'optimal' excitation levels represent the minimum constant excitation levels sufficient to support stable standing, while the 'maximal' excitation levels represent the largest constant excitation levels supporting the same posture. The 'optimal' hip (36.2 N-m) and knee (11.5 N-m) extension moment constraints were selected as those minimally necessary to support stable erect standing in energy efficient postures without joint contractures as reported in [Kagaya et al.] [30]. Joint moment constraints at the trunk (20.2 N-m, [extension]) and ankles (2.9 N-m, PF) were subsequently selected such that the static [UL] loading was zero when the model shoulder positions were at the set point position. For comparison to clinically relevant systems applying supramaximal stimulation, the 'maximal' set of constant excitations were specified as all muscles fully excited (excitation $=1.0$ ) except the ankle plantarflexors, which were adjusted to 0.262 as part of the requirement to minimize static [UL] loading at the set point. The 'maximal' set drove the knees, hips, and trunk slightly $\left(<5^{\circ}\right)$ into hyper-extension, i.e., past set point position defining full extension. Clinically, this is desired and commonly observed" [9].

\section{Procedure for Creating Optimal Muscle Activation Data According to Center of Mass Acceleration Targets}

We employed a model-based procedure to generate the data used to train the ANN in the FNS control system. The procedure determined optimal muscle activation patterns in accordance with specified COM acceleration targets, expressed in Cartesian coordinates with respect to a globally fixed reference frame. We performed the procedure twice, each with a different muscle set. The first muscle set was restricted to the 16 muscle groups with SCI-adjusted force properties targeted for activation by an implanted neuroprosthesis. We used data from this muscle set to train the ANN and develop the FNS controller acting to resist postural disturbances. The second muscle set represented nondisabled function and included all 58 muscle groups available across the trunk and lower limbs without SCI force adjustment. We used results from this muscle set to validate the procedure against EMG data collected for nondisabled subjects undergoing standing disturbances ("Collection of Electromyographic Data of Nondisabled Individuals During Perturbed Bipedal Standing” section). Figure 2 depicts the procedure, which is outlined as follows.

\section{Step 1}

Using the model system equations of motion (SD/ FAST, Symbolic Dynamics, Inc; Mountain View, California), we determined the maximal COM acceleration $\left(a_{C O M}\right)$ induced due to the maximal change in muscle activation for each muscle group (i) with the initial position state at the set point position and zero initial velocity and acceleration for all muscle and skeletal states. The maximal change in muscle activation $\left(\Delta M_{\max }\right)$ is the full 
(a)

Step 1

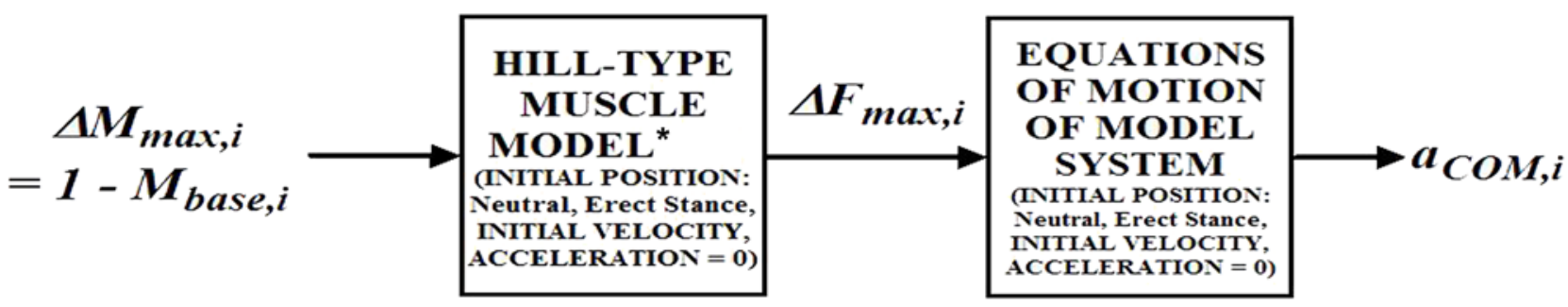

(b)

\section{Step 2}

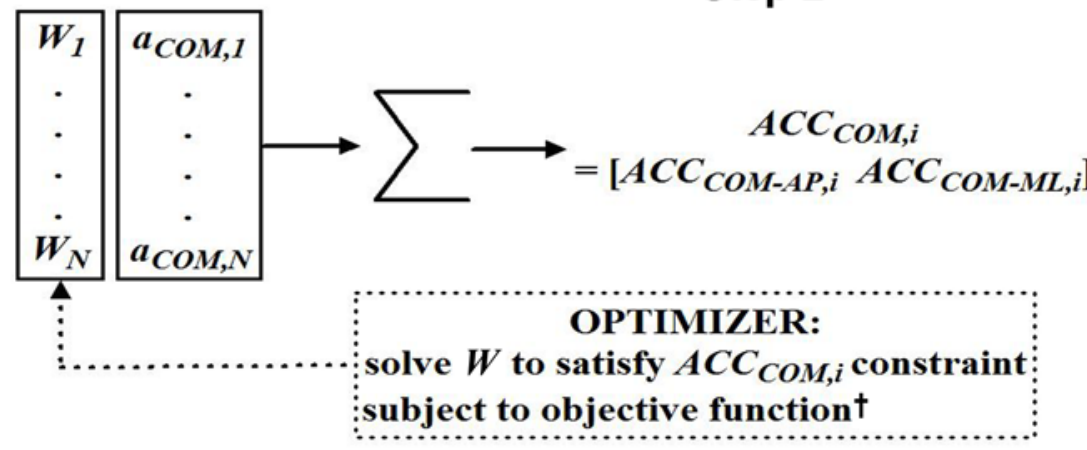

$$
1
$$
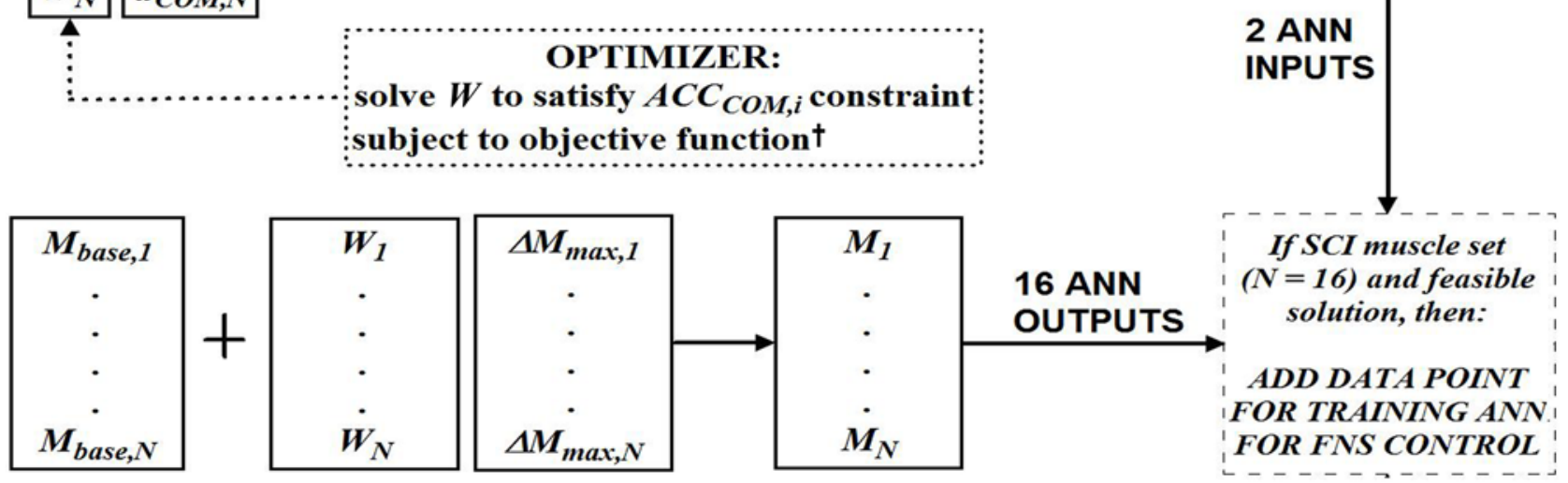

\section{Figure 2.}

Two-step procedure for creating optimal muscle activation data in accordance with center of mass (COM) acceleration targets for training artificial neural network (ANN) of functional neuromuscular stimulation (FNS) controller. (a) Apply maximal change in activation $\left(\Delta M_{\max }\right)$ for muscle group $(i)$ to produce corresponding maximal change in muscle force $\left(\Delta F_{\text {max }}\right)$ with muscle whose initial state corresponds to that during neutral (zero velocity, acceleration), erect standing defined as set point. Model system equations of motion determine corresponding maximum induced acceleration $\left(a_{C O M, i}\right)$ of total-body COM with system again placed at neutral erect set point. (b) Optimization routine determines weighting factors to solve constraint equations defining anterior-posterior and medial-lateral components of given COM acceleration target $\left(A C C_{C O M}\right)$. If feasible solution found, then COM acceleration target and corresponding optimal muscle activations solution is retained as single data point for training ANN. AP = anterior-posterior, $M=$ muscle activation, $M_{\text {base }}=$ base muscle activation, $\mathrm{ML}=$ medial-lateral, $N=$ number of muscle groups, $W_{1}=$ weighting factor for muscle group 1, $W_{N}=$ weighting factor for muscle group $N$. 'Zajac FE. Muscle and tendon: Properties, models, scaling, and application to biomechanics and motor control. Crit Rev Biomed Eng. 1989;17(4):359-411. ${ }^{\dagger}$ Crowninshield RD, Brand RA. A physiologically based criterion of muscle force prediction in locomotion. J Biomech. 1981;14(11):793-801.

activation level (normalized to equal 1) minus the baseline muscle activation level $\left(M_{\text {base }}\right)$ used for steady-state standing maintenance. In the SCI construction, the baseline activation levels were set equal to the "optimal" con- stant excitation set described in the "Determining Optimal Maximal Sets of Constant Excitation Levels for Baseline Performance" section. For the nondisabled case, we assumed that baseline activation of all 58 muscles was 0 , 
since during quiet standing, we observed EMG activity to be negligibly low [14]. We also assumed that tibialis anterior does not produce significant accelerations on the system since its isolated FNS activity produces net ankle DF, resulting in simple lifting of the anterior foot (i.e., toe-off) at neutral stance. Consequently, we removed tibialis anterior from the analysis for the SCI case. We restricted ankle PF activity to soleus force output with other plantar flexors (medial, lateral gastrocnemius) omitted despite being potentially accessible to FNS with a single stimulation channel at the triceps surae.

All three muscle heads of the triceps surae could be included; in this case, however, we noted from pilot experimentation that optimal excitation levels to the ankle PF group were notably smaller compared with other muscle groups. This directly resulted from a lack of targeted musculature that could produce strong anterior shifts in COM acceleration. This includes certain hip and trunk flexors that pitch the body forward not being stimulated and ankle DF expectedly yielding toe-off from erect stance. In turn, the relatively strong posterior COM accelerations that can be induced from erect stance by the entire triceps surae muscle group were not as effectively balanced. Including only soleus essentially desensitized the optimal ankle PF actions to stimulus input, and we observed that this resulted in better overall standing performance (i.e., reduced UL loading).

\section{Step 2}

Using the $a_{C O M}$ values for $\Delta M_{\max }$ of individual muscle groups, we formulated an optimization to determine the optimal muscle activation solutions to produce a given COM acceleration target from the set point stance. Given proportionality between changes in muscle activation forces and the corresponding accelerations induced upon the system, the linear constraint equations to be satisfied by the optimizer yielded the desired net system COM acceleration $\left(A C C_{C O M}\right)$ targets as follows:

$$
\begin{gathered}
A C C_{C O M-A P}=\sum_{i=1}^{N} a_{C O M-A P, i} W_{i} \text { and } \\
A C C_{C O M-M L}=\sum_{i=1}^{N} a_{C O M-M L, i} W_{i},
\end{gathered}
$$

where $W_{i}=$ weighting factor. The net system COM acceleration is defined here by only two components in the AP and ML directions. We assumed the third dimension of COM acceleration (in the inferior-superior direction) to be small enough to be omitted, provided the system does not collapse given sufficient baseline stimulation to produce basic constraints (e.g., knees do not buckle) typical for standing [31]. Each component target represents an optimization constraint equal to the weighted sum of the respective $a_{C O M}$ that can be induced by an individual muscle group from the baseline level. $W_{i}$ is the normalized (0 to 1 ) change in activation from baseline for each muscle group. We only explored positive (i.e., increase) changes in activation from baseline levels since we assumed the baseline levels are fundamentally necessary to maintain basic standing with FNS. This assumption was necessary since this FNS control system is designed to operate about erect stance. With only COM acceleration feedback to modulate stimulation levels, some measure of FNS activation is necessary to maintain the erect set point when no significant accelerations (e.g., quiet standing) are present. Furthermore, without position feedback to produce alternate combinations of activation that can still preserve the basic erect standing configuration, we only considered increases from baseline activations since decreases may overtly compromise the erect configuration about which these COM acceleration targets are being defined.

Using the Optimization Toolbox in MATLAB (MathWorks; Natick, Massachusetts), we determined the solution vectors $(W)$ within the maximum feasible space of COM acceleration targets. The maximum feasible target for each direction was simply the sum of the absolute values of the respective direction of $a_{C O M}$ (listed in Table 2), multiplied by two (given the symmetry of the left and right side muscle groups). These maximum feasible values were $2.10,0.48$, and $1.74 \mathrm{~m} / \mathrm{s}^{2}$ in the posterior, anterior, and lateral directions, respectively. In creating a solution space encompassing these limits, COM acceleration targets were specified between $\pm 1.8 \mathrm{~m} / \mathrm{s}^{2}$ in the $\mathrm{ML}$ direction and between 2.2 and $0.5 \mathrm{~m} / \mathrm{s}^{2}$ in the AP direction at increments of $0.1 \mathrm{~m} / \mathrm{s}^{2}$, yielding a total of 1,036 targets. We optimized the solution vectors $(W)$ according to minimization of an objective criterion developed for locomotion [32]:

$$
\operatorname{Func}(W)=\min \left(\sum_{k=\text { Musclelendex }}^{\# \text { Muscles }} \sigma_{k}^{2}\right) \quad \sigma_{k}=\frac{\text { Force }_{k}}{\text { Area }_{k}} .
$$


Optimization parameters included a maximum of 10,000 iterations, constraint equation tolerance of $0.01 \mathrm{~m} / \mathrm{s}^{2}$ and function tolerance of $0.001 \mathrm{~N}^{2} / \mathrm{m}^{4}$. If the optimizer produced a solution that met the tolerance for both constraint equations for a given $A C C_{C O M}$, then we classified that COM acceleration target solution as "feasible." We only retained feasible solution points for subsequent EMG analysis or ANN training. The two components of $A C C_{C O M}$ served as the inputs and the corresponding 16 absolute muscle activation solutions $\left(M_{i}\right)$ served as the outputs for ANN training.

\section{Collection of Electromyographic Data of Nondisabled Individuals During Perturbed Bipedal Standing}

To validate the general procedure used to create the muscle activation synergy from the "Procedure for Creating Optimal Muscle Activation Data According to Center of Mass Acceleration Targets" section, we calculated the direction of the resultant COM acceleration for which activity was highest for different muscle groups for the model-based synergy and compared it with a similar metric based on the EMG data collected from three nondisabled volunteers undergoing systematic external perturbations while standing. None showed nor reported a history of orthopedic or vestibular problems. We applied perturbations using software developed in LabVIEW (National Instruments Corporation; Austin, Texas) to control electromagnetic linear actuators (STA2506, Copley Controls; Canton, Massachusetts) mounted on customized framing (80/20 Inc; Columbia City, Indiana) rigidly fixed to either floor or wall surfaces. Subjects stood with arms crossed and wore a weight belt approximately at COM level. We positioned it perpendicularly to four actuator complexes placed in front, back, right, and left of the subject. We tied four ropes off on one end onto the belt with each rope connected and directly aligned with the piston of an actuator on the other end. We used a customized aluminum plate with attached rope cleat to quickly fasten, adjust for length, and release the rope from the actuator. All programmed disturbances were discrete force pulses, $250 \mathrm{~ms}$ in duration. We determined the force pulse amplitude threshold that elicited stepping for each subject in each of the four directions by trial and error. Perturbations were limited to 80 percent of the stepping threshold. COM acceleration under these conditions was not a controlled variable but assumed to be close to the maximal values possible during stable bipedal standing and could be interpreted as proportion- ally equal in each direction. We applied 30 perturbations on each subject in each direction.

We recorded EMG signals bilaterally from muscles approximately coincident with those targeted for stimulation in neuroprosthesis recipients: tibialis anterior, soleus, vasti, semimembranosus, gluteus maximus, gluteus medius, adductor magnus, and lower erector spinae. We collected EMG data using disposable, self-adhesive surface electrodes placed according to SENIAM (surface EMG for noninvasive assessment of muscles) standards (http://www.seniam.org). We acquired data with a Telemyo 900 (Noraxon; Scottsdale, Arizona) at a sampling frequency of 1,500 Hz. EMG signals were rectified and band-limited by a $50 \mathrm{~Hz}$ fourth-order low-pass Butterworth filter offline as specified by Krishnamoorthy et al. [15]. We determined the mean amplitude ( $\mathrm{amp}$ ) of the processed EMG during the perturbation period across all trials for each muscle $(j)$ in each direction $(k)$. For each muscle, we calculated the EMG activation vector $\left(\overrightarrow{e m g \_a c t}\right)$, representing which net direction is most active, as follows:

$$
\overrightarrow{e m g \_a c t}=\sum_{k=1}^{4} a m p_{j, k} D_{k}
$$

where $D_{k}=$ a unit-direction vector in the opposite direction of the perturbation and is represented in $x y$-Cartesian coordinates where $\pm x$ correspond to front and back and $\pm y$ correspond to right and left. We used the opposite direction of the pull since we assumed that muscle activity initially increases to resist COM acceleration effects produced by the disturbances. Final activation vectors were unity-normalized for graphical display since we only used net directional information to compare between model and EMG results.

\section{Comparing Nondisabled Elecromyographic Data Against Optimal Model-Based Data}

Using standard conversion of Cartesian to polar coordinates, we calculated the angular coordinate $\left(\theta_{E M G}\right)$ of $\left(\overrightarrow{e m g \_a c t}\right)$ for each muscle to specify the primary direction of activation for each muscle group opposing the systematic perturbations during nondisabled bipedal standing. The polar angular coordinate $\left(\theta_{S Y N}\right)$ serves as the primary direction of activation for each muscle group 
according to the model-based synergy, which we determined from the following activation vector quantity:

$$
\overrightarrow{\text { syn_act }_{j}}=\sum_{k=1}^{N} A C C_{C O M-k} X_{j},
$$

where $X_{j}=$ the corresponding muscle excitation solution from the "Procedure for Creating Optimal Muscle Activation Data According to Center of Mass Acceleration Targets" section. We observed correspondence between the angular coordinates for the EMG and model-based vectors for each muscle group.

\section{Creating Artificial Neural Network for Functional Neuromuscular Stimulation Control}

AP and ML components of each feasible COM acceleration target resulting from the simulations were the inputs and the corresponding 16 optimal muscle excitation levels were the outputs for a single ANN data point. We randomly assigned feasible data points for training (70\%), testing (20\%), and validating (10\%) the ANN. We constructed the ANN with the Neural Network Toolbox in MATLAB. We employed a three-layer (input, hidden, output layers) feedforward ANN structure for its universal mapping capability of nonlinear functions [33]. We determined the number of hidden layer neurons to be 18 by heuristically finding the number of neurons providing the lowest mean squared error after 1,000 training epochs. We normalized all input and output data over $[-1,+1]$ prior to training. The training function was the Levenberg-Marquardt algorithm [34]. A maximum of 10,000 epochs were specified for training in lieu of an early stopping criterion specified as 250 consecutive epochs of increasing fitting error to the validation set. We calculated the ANN output sensitivity as the slope for ANN output excitation in each acceleration direction for each muscle group at neutral stance with zero acceleration input.

\section{Perturbation Simulations}

"In all, 978 perturbation simulations were conducted to optimally tune and evaluate the controller with respect to total [UL] loading. Total [UL] loading was the sum of the 'net' force applied at the left and right shoulders. For each simulation, the computer model started at the desired erect set point, and [UL] loading was tracked during the perturbation and following recovery period (750 ms). This recovery period was sufficient to sustain effective stabilization, defined as [UL] loading within $1 \%$ body weight
(BW) of its final steady-state value, across all simulations. Each perturbation simulation included a single pulse force disturbance applied at a single location. The location, direction, magnitude, and duration of the perturbation were varied with each simulation. Perturbations were applied at the COM locations of the thorax, pelvis, femur, or shank segment in the forward, backward, left, or right directions relative to a globally fixed Cartesian reference frame. These force disturbances ranged from 5\% to $15 \%$ BW in magnitude and 50 to $500 \mathrm{~ms}$ in duration. Perturbations were also repeated at the system COM, also expressed in global three-dimensional coordinates” [9].

\section{Tuning Center of Mass Acceleration Feedback Controller}

For dynamic controller action, we multiplied each of the two acceleration inputs (i) to the ANN by its respective proportional gain $\left(K_{P, i}\right)$ as follows:

$$
A N N_{-} \text {Input }_{i}=K_{P, i} \times A C C_{C O M-i} \cdot
$$

Gains were optimized to minimize the objective function criterion of the total two-arm UL loading necessary for stabilization during perturbation and recovery over all 978 simulations. The gains for both acceleration inputs were optimally tuned using an asynchronous parallel pattern set global search algorithm implemented in the APPSPACK [35] software package running on a FUSION A8 multi-processor computer (Western Scientific, Inc; Valencia, California). We determined algorithm parameters such that solutions were found within $100 \mathrm{~h}$ of computational time. These parameters include initial step size equal to 1 , step tolerance equal to 0.01 , and step contraction factor equal to 0.985 . The gains were bounded between 0 and -10 . The negative value indicates negative feedback whereby the control system acted to produce effects that counter the COM acceleration observed during perturbation and recovery. The initial gain values were based on manual tuning. This process involved stepwise increments of each feedback gain to minimize UL loading while holding the other feedback gain to 0 . The test perturbation for manual tuning was a $100 \mathrm{~N}, 200 \mathrm{~ms}$ force pulse at the thorax. 


\section{Testing Controller Performance}

\section{External Force Pulse Perturbations}

We repeated all 978 perturbation simulations with the feedback controller active and with constant baseline (optimal or maximal) excitation levels under two-arm and one-arm support conditions. We optimally tuned control systems according to two-arm support but tested them under both support conditions to observe general controller performance capabilities, including disturbance rejection, while potentially keeping one arm free for object manipulation. The fundamental synergy defined by the optimal acceleration-excitation data remains unchanged regardless of support condition. Even the net synergy after determination of optimally tuned feedback gains remains symmetric since only one feedback gain is present for each test dimension. Furthermore, we expected that with tuning of a similar FNS control system deployed under live conditions, the subject with SCI would initially resist external perturbations under two-arm support prior to testing with one-arm support while performing functional tasks. Therefore, we found it reasonable to tune under two-arm support conditions and test for both two-arm and one-arm support in simulation. We determined the level of significance of any reduction in UL loading with the controller active compared with baseline across perturbation direction, location, and magnitude by multiple analysis of variance.

\section{Functional Task Performance}

"Functional implications of the controller were assessed in simulation with application of sinusoidal force loads at one shoulder to mimic postural distur- bances due to weighted, voluntary single arm movements. Three-dimensional, sinusoidal force loading was applied at the left shoulder while [UL] control was applied only at the right shoulder (i.e., one-arm support). The applied sinusoid forces were as follows: [AP]: $1 \mathrm{~Hz}$, $10 \mathrm{~N}$ amplitude, $0 \mathrm{~N}$ offset; Right/Left: $1 \mathrm{~Hz}, 20 \mathrm{~N}$ amplitude, $0 \mathrm{~N}$ offset; Superior/Inferior: $0.5 \mathrm{~Hz}, 20 \mathrm{~N}$ amplitude, $-50 \mathrm{~N}$ offset. These amplitude and frequency specifications were consistent with those observed in loaded (2.27 kg) single arm voluntary movements described in [Triolo et al.] [36]" [9].

\section{RESULTS}

\section{Induced Center of Mass Acceleration Results}

Table 2 lists the maximum COM acceleration induced from neutral stance by each SCI muscle group targeted for stimulation. The soleus and gluteus medius produced the largest induced COM accelerations in the posterior direction. This is explained by basic anatomical constraints of the ankles and hips being located below the COM, whereby ankle PF and hip extension in the sagittal plane would drive the system backward. Gluteus medius and adductor magnus induced the largest COM accelerations in the ML dimension. This is also anatomically consistent given their primary articulations of hip abduction and adduction, whose effects were largest in the coronal plane. Gluteus maximus and tibialis anterior produced no changes in COM acceleration since gluteus maximus was already maximally activated at its initial level and tibialis anterior was assumed to produce toe-off at erect stance. Only semimembranosus and erector spinae induced

Table 2.

Maximum acceleration of total body center of mass (COM) induced from quiet erect stance for each muscle group targeted for stimulation.

\begin{tabular}{|c|c|c|c|c|}
\hline $\begin{array}{c}\text { Muscle Group } \\
\text { (Right Side) }\end{array}$ & $\begin{array}{c}\mathrm{SCI} \\
a_{C O M-A P}\left(\mathrm{~m} / \mathrm{s}^{2}\right)\end{array}$ & $\begin{array}{c}\mathrm{SCI} \\
a_{C O M-M L}\left(\mathrm{~m} / \mathrm{s}^{2}\right)\end{array}$ & $\begin{array}{c}\text { Nondisabled } \\
a_{C O M-A P}\left(\mathrm{~m} / \mathrm{s}^{2}\right)\end{array}$ & $\begin{array}{c}\text { Nondisabled } \\
a_{C O M-M L}\left(\mathrm{~m} / \mathrm{s}^{2}\right)\end{array}$ \\
\hline Soleus & -0.559 & 0.011 & -1.587 & 0.030 \\
\hline Tibialis Anterior & NA & NA & 1.699 & -0.398 \\
\hline Vasti & -0.064 & 0.004 & -2.661 & 0.016 \\
\hline Adductor Magnus & -0.034 & 0.307 & -0.265 & 3.494 \\
\hline Gluteus Maximus & 0.000 & 0.000 & -2.077 & -0.640 \\
\hline Semimembranosus & 0.228 & 0.078 & 0.726 & 0.247 \\
\hline Erector Spinae & 0.009 & -0.006 & 0.048 & -0.032 \\
\hline
\end{tabular}

Note: Positive values are either in anterior (front) or right directions.

$a_{C O M}=$ COM acceleration induced by maximal change in activation of given muscle group, $\mathrm{AP}=$ anterior-posterior, $\mathrm{ML}=$ medial-lateral, $\mathrm{NA}=$ not applicable, $\mathrm{SCI}=$ spinal cord injury. 
COM accelerations in the anterior direction. While these muscles extend the hip and trunk, respectively, they both generate forward motion of the pelvis and lower torso. Anatomical constraints explain this with semimembranosus producing knee flexion in conjunction with hip extension and the erector spinae spanning the entire mid-tolower torso. Prolonged activity of these extensor muscles may drive the system posteriorly, but their instantaneous effects from quiet, neutral standing is to shift the COM anteriorly. Vasti and erector spinae effects were small, relative to other muscles. The vasti were nearly maximally activated at baseline, and erector spinae did not produce a very significant instantaneous acceleration at neutral standing.

\section{Electromyographic Validation of Center of Mass Acceleration Mapping}

Figure 3 shows the primary activation directions for each right side muscle group from both the nondisabled EMG data set and the nondisabled model synergy. The methodology for EMG collection and analysis was robust because the standard deviation (SD) about the mean $\theta_{E M G}$ for all muscle groups was $<20^{\circ}$, indicating that \pm 2 SD are encompassed within a single quadrant of the twodimensional direction space. The model polar angular coordinate $\left(\theta_{S Y N}\right)$ was within the quadrant centered about $\theta_{E M G}$ for all muscle groups except vasti, semimembranosus, and gluteus maximus. These exceptions in $\theta_{E M G}$ can be attributed to the positional stabilization required to prevent collapse during perturbations applied at the lower torso in the live-subject experiments. Specifically, the increases in EMG activity for semimembranosus and vasti were likely necessary to prevent hip and knee flexion induced by forward and backward disturbances, respectively. Higher gluteus maximus EMG against backward disturbances may be explained by a co-contraction response in conjunction with antagonist muscles to generally stiffen the hips. For the other five muscle groups, good correspondence in activation directions indicate that their first-response contributions to stabilize standing can be described in accordance with the initial COM acceleration direction induced by a perturbation from quiet bipedal standing.

\section{Artificial Neural Network Results}

The ANN was capable of accurately outputting the synergistic muscle excitation patterns optimized according to COM acceleration input targets. The mean errors

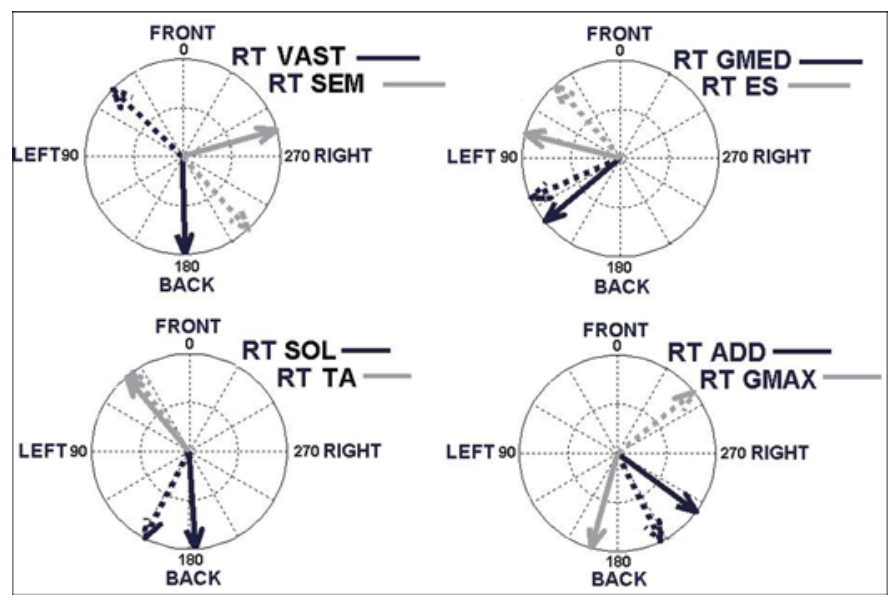

Figure 3.

Compass diagrams displaying theoretical "net activation directions" for various muscle groups. Net activation direction suggests muscle group is most active when system center of mass is being accelerated in that direction as result of all muscles activating to directly counter (i.e., oppose) disturbance from neutral standing. Activation direction $(\theta)$ results displayed for respective nondisabled model (solid arrow) and electromyographic (broken arrow) data for following right-side (RT) muscle groups: soleus (SOL, $\theta=183^{\circ}, 153^{\circ} \pm 13^{\circ}$ ), tibialis anterior (TA, $\theta=40^{\circ}, 35^{\circ} \pm 11^{\circ}$ ), vastus intermedius (VAST, $\theta=181^{\circ}, 46^{\circ} \pm$ $13^{\circ}$ ), semimembranosus (SEM, $\theta=286^{\circ}, 222^{\circ} \pm 12^{\circ}$ ), adductor magnus (ADD, $\theta=234^{\circ}, 207^{\circ} \pm 13^{\circ}$ ), gluteus maximus (GMAX, $\theta=164^{\circ}, 319^{\circ} \pm 17^{\circ}$ ), gluteus medius (GMED, $\theta=130^{\circ}, 114^{\circ} \pm$ $19^{\circ}$ ), erector spinae (ES, $\theta=75^{\circ}, 41^{\circ} \pm 12^{\circ}$ ).

(Table 3) in outputs by the ANN for all feasible COM acceleration target inputs were $<0.001$. This demonstrates that the ANN was an effective structure to represent the synergy that is to be driven by feedback control in forward simulations. Figure 4 shows the ANN excitation surface outputs for five right-side muscle groups. Note that tibialis anterior was omitted because of potential toe-off, and gluteus maximus and vasti were nearly maximally activated simply to meet the specified optimal baseline requirements for standing. The output surfaces indicate that the soleus is prominent in accelerating the system COM backward across the entire feasible target space. The right semimembranosus has observable increased activity in accelerating the COM forward. The muscle groups most active in driving the system COM in the right and left directions were the right adductor 
JRRD, Volume 49, Number 2, 2012

Table 3.

Artificial neural network (ANN) excitation output results: sensitivity at zero acceleration and mean ANN prediction error.

\begin{tabular}{lccc}
\hline \multicolumn{1}{c}{$\begin{array}{c}\text { Muscle Group } \\
\text { (Right) }\end{array}$} & $\frac{\partial e x c i t}{\partial a_{\text {COM }-A P}}$ & $\frac{\partial e x c i t}{\partial a_{\text {CoM }-M L}}$ & $\begin{array}{c}\text { Mean Error in ANN Output } \\
\text { (Excitation) }\end{array}$ \\
\hline Soleus & -0.415 & 0.000 & $1.57 \mathrm{e}-4$ \\
Tibialis Anterior & $\mathrm{NA}$ & $\mathrm{NA}$ & $\mathrm{NA}$ \\
Vasti & 0.000 & 0.000 & $<1 \mathrm{e}-5$ \\
Adductor Magnus & -0.006 & 0.151 & $2.47 \mathrm{e}-4$ \\
Gluteus Maximus & $\mathrm{NA}$ & $\mathrm{NA}$ & $\mathrm{NA}$ \\
Gluteus Medius & -0.047 & -0.330 & $1.84 \mathrm{e}-4$ \\
Semimembranosus & 1.095 & 0.172 & $3.94 \mathrm{e}-4$ \\
Erector Spinae & 0.000 & 0.000 & $5.27 \mathrm{e}-5$ \\
\hline
\end{tabular}

Note: Not applicable (NA) for muscle groups either not targeted for feedback control or always fully activated.

$a_{C O M}=\mathrm{COM}$ acceleration induced by maximal change in activation of given muscle group, $\mathrm{AP}=$ anterior-posterior, excit $=$ muscle excitation level, $\mathrm{ML}=$ mediallateral.
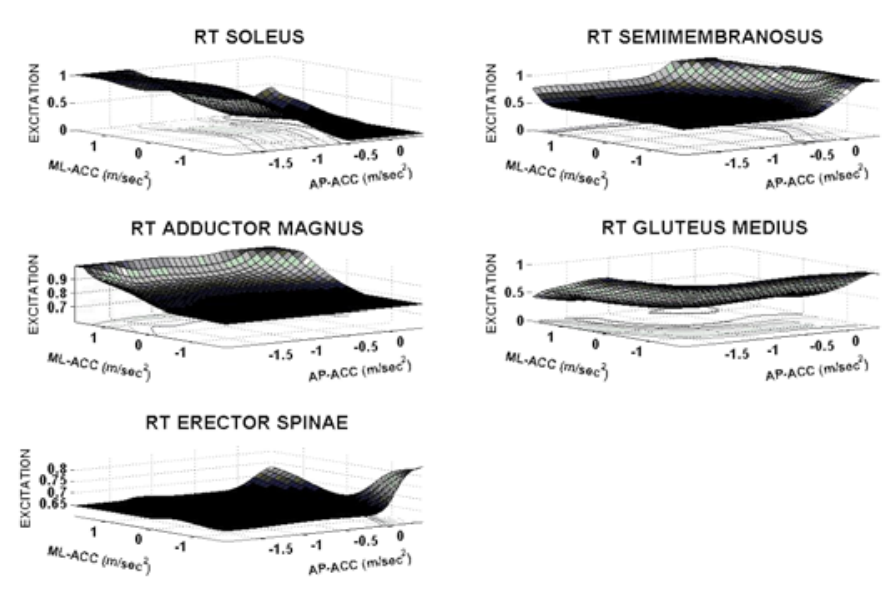

Figure 4.

Artificial neural network excitation output of select right-side (RT) muscles as function of center of mass acceleration inputs. Note: positive axes $=$ anterior, right. $\mathrm{ACC}=$ acceleration, $\mathrm{AP}=$ anteriorposterior, $\mathrm{ML}=$ medial-lateral.

magnus and right gluteus medius, respectively. This follows anatomical intuition with the ANN providing smooth output of the optimization space used for ANN training. Sensitivity results in Table 3 suggest that all targeted muscle groups in Figure 4, except erector spinae, would be recruited immediately in disturbance rejection as they undergo notable recruitment to even small (near zero-acceleration point) changes in acceleration. Erector spinae would be additionally recruited only with sufficiently increased AP and ML acceleration.

\section{Controller Gain Tuning}

The final feedback gains that minimized total UL loading during external perturbations were $-5.17 \mathrm{e}-2$ and -0.99 for the AP and ML COM acceleration component inputs, respectively. The higher allowable feedback gain for the ML component can be explained by greater inherent stability of bipedal standing in that direction. The BOS is wider in the ML direction and the standing system approximates a 4-bar linkage [37] as compared with a more unstable inverted pendulum in the AP direction [38].

\section{Controller Performance}

Figure 5 shows typical two-arm UL loading and muscle-induced joint moments for baseline and controlleractive conditions. In response to 15 percent BW, $250 \mathrm{~ms}$ force pulses applied at the model COM in the AP and lateral directions, the controller reduced total UL loading during the perturbation and recovery periods by 43 and 66 percent, respectively. The controller provided robust return to the set point posture with near zero final UL loading. The joint moments produced by the controller during steady-state before and after the perturbation were lower than maximal baseline as expected and identical to optimal baseline as designed. The robust return to optimal baseline performance indicates that acceleration feedback control was transient because the controller did not produce instabilities requiring excessive UL loading. The peak UL loading produced with controller action was below that with maximum baseline stimulation in both perturbation directions.

Consistent with anatomical function, ankle PF and hip extension were prominent in resisting a forward 


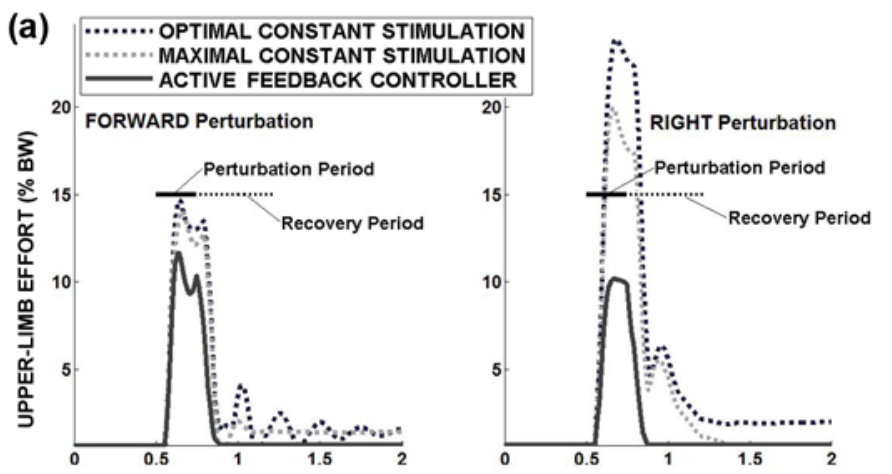

(b)
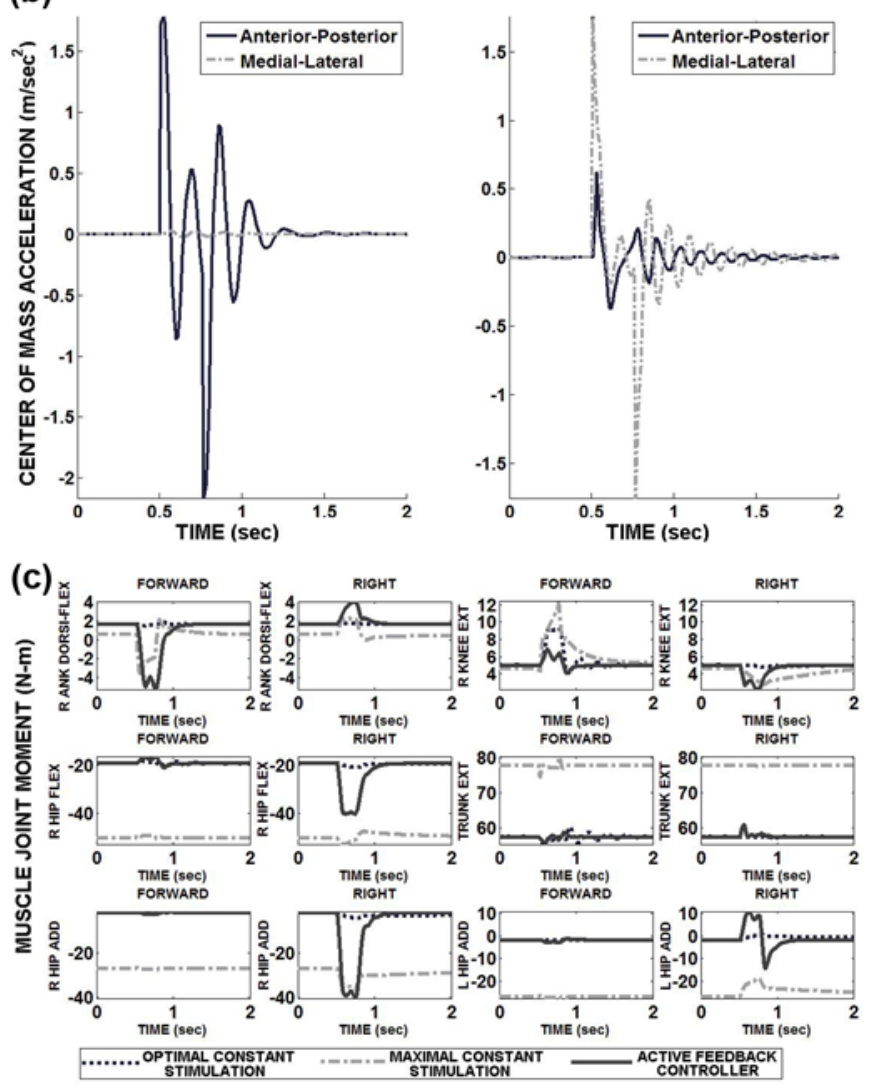

Figure 5.

(a) Two-arm upper-limb loading, (b) center of mass (COM) acceleration feedback, and (c) muscle-induced joint moments to stabilize against perturbation pulse (15\% body weight, $250 \mathrm{~ms})$ applied at model COM in either forward or side (i.e., right) direction. Add $=$ adduction, Ank $=$ ankle, $\mathrm{BW}=$ body weight, Dorsiflex $=$ dorsiflexion, Flex $=$ flexion, $L=$ left, $R=$ right-side.

disturbance. Correspondingly, right hip abduction and left hip adduction were strongly activated to reject the rightward disturbance. Trunk extension was small even against a forward disturbance since it was applied at the system COM, which is too low to produce significant trunk flexion. Knee extension moments were small in all cases despite high vasti excitation because the knees were generally held in hyperextension where length-tension properties limited force output. The largest ( $>5 \mathrm{~N}-\mathrm{m})$ controller-mediated changes in joint moments occurred at the ankles and hips, reflecting the well-described ankle and hip strategies for stable standing [14].

We observed smooth UL loading and joint moment profiles with controller feedback despite some oscillations (Figure 5(b)) in the COM acceleration feedback signal, indicating that the mass-inertia of the system and delays in muscle force actuation were able to sufficiently dampen those effects. For a sideward perturbation, ML acceleration was more prominent as expected, but the AP acceleration component was still notable. This further underscores how sensitive the SCI standing system is to destabilizing effects in the AP direction compared with the ML direction.

Table 4 shows composite simulation results for onearm and two-arm resistance to perturbations. Maximal constant excitation always resulted in lower UL loading than optimal, but the acceleration feedback controller improved performance over baseline case in all listed condition cases except backward perturbations. This results from only semimembranosus being available to induce forward COM accelerations from neutral stance. UL loading also increased as perturbations were applied to more superiorly located segments. Perturbations applied to lower segments were more attenuated by muscle and inertial effects before greater UL stabilization was required. UL loading is significantly greater (i.e., standing is more unstable) during one-arm support under optimal or maximal baseline stimulation. Optimal baseline stimulation was further ineffective in one-arm support because the model COM occasionally failed to return to within $0.1 \mathrm{~m}$ of its original position. With the ANN controller active, similar UL loading was expended in resisting perturbations with either one-arm (21 N) or two-arm (20 N) support, demonstrating the consistency and value of feedback control. The mean reduction in UL loading with the controller active compared with maximal baseline across all force pulse perturbations over both one-arm and two-arm conditions was 43 percent. The controller produced a statistically significant reduction in UL loading with rejection of the null hypothesis of equal means at $p=0.05$ across all perturbation variables (direction, location, amplitude) compared 
JRRD, Volume 49, Number 2, 2012

Table 4.

Upper-limb (UL) loading for stabilization against postural disturbances.

\begin{tabular}{|c|c|c|c|c|}
\hline \multirow[t]{2}{*}{ Disturbance Condition } & \multicolumn{2}{|c|}{$\begin{array}{l}\text { Mean Baseline } \\
\text { UL Loading (N) }\end{array}$} & \multirow{2}{*}{$\begin{array}{l}\text { Mean Controller } \\
\text { UL Loading (N) }\end{array}$} & \multirow{2}{*}{$\begin{array}{c}\text { Reduction with Controller } \\
\text { (\%) }\end{array}$} \\
\hline & Optimal & Maximal & & \\
\hline \multicolumn{5}{|l|}{ Direction } \\
\hline Forward & 66 & 47 & 27 & 44 \\
\hline Backward & 32 & 22 & 25 & -14 \\
\hline Side (Left or Right) & 51 & 36 & 14 & 61 \\
\hline Pelvis & 58 & 41 & 20 & 49 \\
\hline Thigh (Left or Right) & 52 & 35 & 19 & 44 \\
\hline Shank (Left or Right) & 39 & 22 & 8 & 63 \\
\hline \multicolumn{5}{|l|}{ Support Conditions } \\
\hline Two-Arm Support & 32 & 28 & 20 & 29 \\
\hline
\end{tabular}

with baseline. During one-arm functional task performance (FTP), the controller kept the model erect and reduced UL loading by 51 percent compared with maximal baseline excitation.

\section{DISCUSSION}

To address inherent drawbacks to joint feedback, we proposed and evaluated the feasibility of COM acceleration feedback for control of FNS standing. Using a threedimensional model of bipedal SCI standing, we developed a control system using COM acceleration feedback to modulate muscle excitation levels and reduce the UL loading required to stabilize against postural disturbances. Use of COM acceleration as a feedback signal follows directly from previous studies that have implicated acceleration [10-12] and COM dynamics [13] in standing balance control. In this study, we demonstrated that COM acceleration is a potentially valuable feedback parameter for characterizing standing control specifically against perturbations.

We outlined a methodology to produce an optimal synergy that relates changes in muscle activation from neutral standing to changes in COM acceleration using our anatomically realistic model. We validated the resultant synergy by comparing which net direction certain muscle groups were most active to accelerate the system
COM in opposing a disturbance for a nondisabled model synergy against EMG measurements recorded from the same muscle groups during systematic perturbation of nondisabled standing subjects. Five muscle groups (tibialis anterior, soleus, gluteus medius, adductor magnus, and lower erector spinae) demonstrated high correspondence between the model-constructed synergy and live EMG data. This indicates that these muscle groups should be consistently targeted for FNS control under COM acceleration feedback to stabilize against disturbances.

However, the remaining three muscle groups (gluteus maximus, vasti, and semimembranosus) did not correspond well. We postulated that activity of these muscle groups was modulated according to positional requirements for maintaining erect stance that are not considered in the construction of the activation-acceleration map. Thus, it may be best to reserve these muscle groups for position-based feedback or simply constant activation for basic standing support. In fact, these same muscle groups had relatively high baseline activation levels determined as optimal for sufficiently stable SCI standing (Table 1) and have also been commonly targeted for stimulation to provide basic standing support during clinical application [1]. For FNS control of standing that utilizes feedback of only COM acceleration, position-based corrections would need to be made volitionally by the user but assisted dynamically by modulation of stimulation levels 
for the muscles not reserved for basic standing support such that user effort was minimized.

For forward simulations of FNS feedback control, we employed the same activation-acceleration mapping procedure to construct another model-based synergy using only muscle groups targeted by a 16-channel implant [23] and reflecting typical FNS force-generating capabilities following SCI [24]. In simulation, this SCI-specific synergy was represented by an ANN driven by proportional COM acceleration feedback, which was more than capable of effectively mapping this synergy between only two COM acceleration inputs and 16 muscle excitation outputs. With a prediction error $<1 \mathrm{e}-3$ for all outputs, the ANN was successfully driven by proportional feedback to modulate excitation levels for reducing UL loading required to resist disturbances compared with the typical clinical case of maximal constant muscle excitation. COM acceleration feedback control markedly reduced UL loading across all external disturbances for both two-arm and one-arm support conditions by 43 percent and during FTP by 51 percent. Disturbance rejection during one-arm support and FTP are conditions more pertinent to standing activities of daily living. Thus, future investigations may include optimizing the control system with one-arm support. However, results from this study demonstrate similar total UL loading with the controller active regardless of support condition, suggesting the robustness of controller action despite the nature of support.

The previous paragraph demonstrates the potential of COM acceleration feedback to provide a notable improvement in neuroprosthetic standing performance despite the limited number of paralyzed muscles available. The same muscles were required to both support the body against collapse and generate the additional moments required to reject perturbations. While basic upright support was achieved with optimal baseline stimulation levels generating necessary joint moments as reported by Kagaya et al. [30], gluteus maximus and vasti were nearly maximally activated to produce the necessary baseline hip and knee extension moments and could not be recruited to resist disturbances. We also omitted tibialis anterior from the study. Yet recruitment of the remaining 10 muscles was still sufficient to produce an effective balance control system in simulation, further highlighting the potential benefits of COM acceleration feedback.

The formulation of the controller presented in this study is based on proportional feedback driving an ANN that imposed a synergy to generate optimal changes in muscle activation to produce desired changes in COM acceleration and counter effects of postural disturbance about neutral, erect stance. We employed negative feedback to recruit the muscles required to oppose the COM accelerations encountered during perturbation and recovery. We validated this construction by observing reductions in upper-body loading with simulated one-arm and two-arm support during a wide range of disturbance locations, amplitudes, and directions, as well as during simulated functional tasks with our SCI-adjusted model. Furthermore, our nondisabled EMG data corroborated this approach and coincided with simulation results indicating that muscle groups are largely activated to counter the disturbances reflected in COM acceleration direction. We consistently observed model-predicted COM accelerations to be in the opposite direction of the net action of the most active muscles during repeated disturbances. Notable exceptions (vasti and gluteus maximus) can be attributed primarily to muscle recruitment for other objectives, such as the necessary positional corrections to prevent frank system failure and outright falls. This underlies the notion that comprehensive standing is a complex, multisensory task [39] that employs joint-based feedback. Theoretically, some form of position or joint-based control would be required to replicate the intact balance control apparatus and achieve truly hands-free standing with FNS. However, coordination of muscle activity according to COM acceleration still seems to characterize much of the initial standing response to applied disturbances. More importantly, this synergy may be exploited for substantially extending and improving the functionality of standing neuroprostheses.

This approach for constructing a muscle-based acceleration synergy for neuroprosthetic standing was inspired by Kuo and Zajac [31], who developed an algorithm to generate feasible acceleration sets (FAS) composed of joint angular accelerations for all feasible normalized muscle activations subject to observed experimental constraints (e.g., knees-locked, heel and toe lift-off) of sagittal plane standing. That study used the FAS to identify which muscles, if strengthened, would produce the greatest increases in standing mobility. Our study created feasible, optimal activation patterns that could generate targeted changes in linear COM acceleration, the proposed sensor-based feedback variable for control of SCI standing. 
Although this article supports the use of COM acceleration as a potentially effective feedback variable controlling standing with FNS, additional work is still required to implement such a control system clinically. The clinical viability of acceleration-based feedback control depends on performance in the presence of typical sources of feedback error (e.g., sensor placement, measurement accuracy, soft tissue effects). The model system presented serves as an appropriate test-bed for future studies to systematically introduce feedback error and quantify its effects on performance. Most importantly, efficient techniques need to be developed to fit, tune, and specify system parameters for a particular user in a clinical setting. Methods have been previously outlined for determining user-specific musculoskeletal and UL controller parameters to develop a model-based system for initial controller tuning and evaluation prior to laboratory implementation [9]. While this study employed a generic bipedal model of standing to conceptually validate the proposed control system producing a potentially substantial improvement in standing performance, creation of user-specific models would be necessary to develop model-based solutions for control systems for specific users. This would include scaling the muscle geometry, muscle force-generating capabilities, and length and massinertia properties of segments. Only an accurate description of those features would generate an optimal control solution that produces kinematic and kinetic responses in simulation that appropriately represent those expected to be observed during live laboratory performance.

The methods from Nataraj et al. for creating userspecific control systems were suggested for a joint feedback system composed of PD inputs from nine individual joints [9]. Properly assessing performance to robustly tune a system with that many feedback gains during live conditions may be intractable. Both model and laboratory development of FNS control systems can be expedited with control structures containing fewer feedback variables. The control system examined in this article employs only two feedback variables (AP and ML COM acceleration) that would need to be measured and two feedback gains that would need to be tuned.

While a user-specific model-based solution could still be explored, a paradigm could also be devised to produce the data (Equations (2) and (3)) used to construct the optimal synergy entirely from a live user. The subject with paralysis could be at neutral erect stance in a walker while sufficient but minimal constant stimulation is applied as the "baseline activation." Stimulation could then be discretely ramped from baseline levels for individual muscles $\left(W_{i}\right)$ and the corresponding peak induced COM acceleration $\left(a_{C O M, i}\right)$ could be recorded. This peak would serve only as an estimate of the $a_{C O M, i}$ induced by a particular muscle group since it would occur across the excitation-activation coupling dynamics, and small postural changes away from the neutral set point position may occur. However, the extent to which the assumption of linear superposition governing Equations (2) and (3) breaks down should be investigated experimentally. Given that significant changes in acceleration can occur without large changes in configuration, the suggested methodology could realistically produce a viable solution for neuroprosthetic standing. Finally, an instrumented walker employing load-sensitive handles could be used to tune the system online against disturbances applied by the perturbation system described in the "Collection of Electromyographic Data of Nondisabled Individuals During Perturbed Bipedal Standing” section, as well as provide a metric of goodness of fit for a clinical system.

\section{CONCLUSIONS}

COM acceleration may be an advantageous alternative to joint kinematics as a feedback variable for control of FNS standing. This study suggests that even with control of only 16 SCI-paralyzed muscles available to provide basic standing support, significant improvement in disturbance rejection can still be achieved with COM acceleration feedback modulation of muscle excitation levels. Further study to demonstrate potential clinical viability is necessary, such as evaluating performance robustness in the presence of expected feedback measurement errors. Ultimately, control systems should be developed according to user-specific characteristics for laboratory testing with live subjects with SCI.

\section{ACKNOWLEDGMENTS}

Author Contributions:

Intellectual conceptualization: R. Nataraj, R. F. Kirsch. Intellectual support and guidance: M. L. Audu, R. J. Triolo. Data collection: R. Nataraj, M. L. Audu.

Data analysis: R. Nataraj.

Drafting of manuscript: R. Nataraj.

Critical revision of manuscript for important intellectual content:

M. L. Audu, R. J. Triolo.

Financial support: R. J. Triolo. 
Financial Disclosures: The authors have declared that no competing interests exist.

Funding/Support: This material was based on work supported by the National Institutes of Health (grant R01 NS040547-04A2) and the Motion Study Laboratory, Louis Stokes Cleveland Department of Veterans Affairs Medical Center.

Additional Contributions: Mr. Nataraj is now with the Biomedical Engineering Department, Cleveland Clinic, Cleveland, Ohio.

Institutional Review: All nondisabled subjects signed informed consent forms approved by the institutional review board of the Louis Stokes Cleveland Department of Veterans Affairs Medical Center.

Participant Follow-up: The authors do not plan to inform participants of the publication of this study. However, participants have been encouraged to contact the laboratory regarding status of the study and related publications.

\section{REFERENCES}

1. Triolo RJ, Bogie K. Lower extremity applications of functional neuromuscular stimulation after spinal cord injury. Top Spinal Cord Inj Rehabil. 1999;5(1):44-65.

2. Kralj A, Bajd T, Turk R. Electrical stimulation providing functional use of paraplegic patient muscles. Med Prog Technol. 1980;7(1):3-9. PMID:6966735

3. Jaeger RJ. Design and simulation of closed-loop electrical stimulation orthoses for restoration of quiet standing in paraplegia. J Biomech. 1986;19(10):825-35.

PMID:3782165

http://dx.doi.org/10.1016/0021-9290(86)90133-8

4. Moynahan M, Chizeck HJ. Characterization of paraplegic disturbance response during FNS standing. IEEE Trans Rehabil Eng. 1993;1(1):43-48.

http://dx.doi.org/10.1109/86.242407

5. Chizeck HJ, Kobetic R, Marsolais EB, Abbas JJ, Donner $\mathrm{IH}$, Simon E. Control of functional neuromuscular stimulation systems for standing and locomotion in paraplegics. Proc IEEE Inst Electr Electron Eng. 1988;76(9):1155-65 http://dx.doi.org/10.1109/5.9661

6. Abbas JJ, Chizeck HJ. Feedback control of coronal plane hip angle in paraplegic subjects using functional neuromuscular stimulation. IEEE Trans Biomed Eng. 1991;38(7): 687-98. PMID:1879862 http://dx.doi.org/10.1109/10.83570

7. Hunt KJ, Gollee H, Jaime RP. Control of paraplegic ankle joint stiffness using FES while standing. Med Eng Phys. 2001;23(8):541-55. PMID:11719077 http://dx.doi.org/10.1016/S1350-4533(01)00089-3

8. Matjaci Z, Bajd T. Arm-free paraplegic standing-Part II: Experimental results. IEEE Trans Rehabil Eng. 1998; 6(2):139-50. PMID:9631321 http://dx.doi.org/10.1109/86.681179
9. Nataraj R, Audu ML, Kirsch RF, Triolo RJ. Comprehensive joint feedback control for standing by functional neuromuscular stimulation-A simulation study. IEEE Trans Neural Syst Rehabil Eng. 2010;18(6):646-57. PMID:20923741 http://dx.doi.org/10.1109/TNSRE.2010.2083693

10. Mayagoitia RE, Lötters JC, Veltink PH, Hermens H. Standing balance evaluation using a triaxial accelerometer. Gait Posture. 2002;16(1):55-59. PMID:12127187 http://dx.doi.org/10.1016/S0966-6362(01)00199-0

11. Moe-Nilssen R, Helbostad JL. Trunk accelerometry as a measure of balance control during quiet standing. Gait Posture. 2002;16(1):60-68. PMID:12127188 http://dx.doi.org/10.1016/S0966-6362(01)00200-4

12. Betker AL, Moussavi ZM, Szturm T. Center of mass approximation and prediction as a function of body acceleration. IEEE Trans Biomed Eng. 2006;53(4):686-93. PMID:16602575 http://dx.doi.org/10.1109/TBME.2006.870222

13. Pai YC, Patton J. Center of mass velocity-position predictions for balance control. J Biomech. 1997;30(4):347-54. PMID:9075002 http://dx.doi.org/10.1016/S0021-9290(96)00165-0

14. Horak FB, Nashner LM. Central programming of postural movements: Adaptation to altered support-surface configurations. J Neurophysiol. 1986;55(6):1369-81. PMID:3734861

15. Krishnamoorthy V, Goodman S, Zatsiorsky V, Latash ML. Muscle synergies during shifts of the center of pressure by standing persons: Identification of muscle modes. Biol Cybern. 2003;89(2):152-61. PMID:12905043 http://dx.doi.org/10.1007/s00422-003-0419-5

16. Winter DA. Biomechanics and motor control of human movement. 2nd ed. New York (NY): Wiley; 1990.

17. Zajac FE, Gordon ME. Determining muscle's force and action in multi-articular movement. Exerc Sport Sci Rev. 1989;17:187-230. PMID:2676547

18. Zajac FE. Muscle and tendon: Properties, models, scaling, and application to biomechanics and motor control. Crit Rev Biomed Eng. 1989;17(4):359-411. PMID:2676342

19. Zhao W, Kirsch RF, Triolo RJ, Delp S. A bipedal, closedchain dynamic model of the human lower extremities and pelvis for simulation-based development of standing and mobility neuroprostheses. Proceedings of the 20th Annual International Conference of the IEEE Engineering in Medicine and Biology Society; 1998 Oct 28-Nov 1; Hong Kong, China. Proc IEEE EMBS. 1998;5(28):2605-8.

20. Lambrecht JM, Audu ML, Triolo RJ, Kirsch RF. Musculoskeletal model of trunk and hips for development of seatedposture-control neuroprosthesis. J Rehabil Res Dev. 2009;46(4):515-28. PMID:19882486 http://dx.doi.org/10.1682/JRRD.2007.08.0115 
21. Amankwah K, Triolo RJ, Kirsch R. Effects of spinal cord injury on lower-limb passive joint moments revealed through a nonlinear viscoelastic model. J Rehabil Res Dev. 2004;41(1):15-32. PMID:15273894 http://dx.doi.org/10.1682/JRRD.2004.01.0015

22. Kim JY, Mills JK, Vette AH, Popovic MR. Optimal combination of minimum degrees of freedom to be actuated in the lower limbs to facilitate arm-free paraplegic standing. J Biomech Eng. 2007;129(6):838-47. PMID:18067387 http://dx.doi.org/10.1115/1.2800767

23. Bhadra N, Kilgore KL, Peckham PH. Implanted stimulators for restoration of function in spinal cord injury. Med Eng Phys. 2001;23(1):19-28. PMID:11344004 http://dx.doi.org/10.1016/S1350-4533(01)00012-1

24. Kobetic R, Marsolais EB. Synthesis of paraplegic gait with multichannel functional neuromuscular stimulation. IEEE Trans Rehabil Eng. 1994;2(2):66-79. http://dx.doi.org/10.1109/86.313148

25. Ogata K. Modern control engineering. 4th ed. Upper Saddle River (NJ): Prentice Hall; 2001.

26. Pai YC, Rogers MW, Patton J, Cain TD, Hanke TA. Static versus dynamic predictions of protective stepping following waist-pull perturbations in young and older adults. J Biomech. 1998;31(12):1111-18. PMID:9882043 http://dx.doi.org/10.1016/S0021-9290(98)00124-9

27. Kandel E, Schwartz JH, Jessell TM. Principles of neural science. 4th ed. New York (NY): McGraw-Hill; 2000.

28. Uhlir JP, Triolo RJ, Davis JA Jr, Bieri C. Performance of epimysial stimulating electrodes in the lower extremities of individuals with spinal cord injury. IEEE Trans Neural Syst Rehabil Eng. 2004;12(2):279-87. PMID:15218941 http://dx.doi.org/10.1109/TNSRE.2004.827224

29. Audu ML, Kirsch RF, Triolo RJ. Experimental verification of a computational technique for determining ground reactions in human bipedal stance. J Biomech. 2007;40(5): 1115-24. PMID:16797023 http://dx.doi.org/10.1016/j.jbiomech.2006.04.016

30. Kagaya H, Sharma M, Kobetic R, Marsolais EB. Ankle, knee, and hip moments during standing with and without joint contractures: Simulation study for functional electrical stimulation. Am J Phys Med Rehabil. 1998;77(1):49-54, quiz 65-66. PMID:9482379 http://dx.doi.org/10.1097/00002060-199801000-00009

31. Kuo AD, Zajac FE. A biomechanical analysis of muscle strength as a limiting factor in standing posture. J Biomech. 1993;26(Suppl 1):137-50. PMID:8505348 http://dx.doi.org/10.1016/0021-9290(93)90085-S

32. Crowninshield RD, Brand RA. A physiologically based criterion of muscle force prediction in locomotion. J Bio- mech. 1981;14(11):793-801. PMID:7334039

http://dx.doi.org/10.1016/0021-9290(81)90035-X

33. Haykin SS. Neural networks: A comprehensive foundation. 2nd ed. London (England): Prentice Hall; 1999.

34. Hagan MT, Menhaj MB. Training feedforward networks with the Marquardt algorithm. IEEE Trans Neural Netw. 1994;5(6):989-93. PMID:18267874 http://dx.doi.org/10.1109/72.329697

35. Gray GA, Kolda TG. Algorithm 856: APPSPACK 4.0: Asynchronous parallel pattern search for derivative-free optimization. ACM Trans Math Softw. 2006;32(3):486-507.

36. Triolo RJ, Werner KN, Kirsch RF. Modeling the postural disturbances caused by upper extremity movements. IEEE Trans Neural Syst Rehabil Eng. 2001;9(2):137-44. PMID:11474966 http://dx.doi.org/10.1109/7333.928573

37. Rietdyk S, Patla AE, Winter DA, Ishac MG, Little CE; North American Congress on Biomechanics. NACOB presentation CSB New Investigator Award. Balance recovery from mediolateral perturbations of the upper body during standing. J Biomech. 1999;32(11):1149-58. PMID:10541064 http://dx.doi.org/10.1016/S0021-9290(99)00116-5

38. Winter DA, Patla AE, Prince F, Ishac M, Gielo-Perczak K. Stiffness control of balance in quiet standing. J Neurophysiol. 1998;80(3):1211-21. PMID:9744933

39. Allum JH, Honegger F. Interactions between vestibular and proprioceptive inputs triggering and modulating human balance-correcting responses differ across muscles. Exp Brain Res. 1998;121(4):478-94. PMID:9746156 http://dx.doi.org/10.1007/s002210050484

Submitted on December 13, 2010. Accepted in revised form on May 16, 2011.

This article and all supplementary material should be cited as follows:

Nataraj R, Audu ML, Kirsch RF, Triolo RJ. Center of mass acceleration feedback control for standing by functional neuromuscular stimulation: A simulation study. J Rehabil Res Dev. 2012;49(2):279-96.

http://dx.doi.org/10.1682/JRRD.2010.12.0235

ResearcherID: Raviraj Nataraj: G-4535-2010

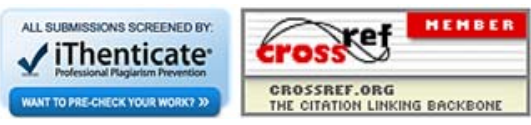

